\title{
Serum HE4 levels combined with CE CT imaging improve the management of monitoring women affected by epithelial ovarian cancer
}

\author{
LUCIA MANGANARO $^{1 *}$, SIMONA MICHIENZI ${ }^{2 *}$, VALERIA VINCI $^{1}$, RENATO FALZARANO $^{2}$, \\ MATTEO SALDARI ${ }^{1}$, TERESA GRANATO ${ }^{3}$, VALENTINA VIGGIANI ${ }^{2}$, \\ LUIGI FRATI $^{2}$ and EMANUELA ANASTASI ${ }^{2}$ \\ Departments of ${ }^{1}$ Radiological, Oncological and Anatomopathological Sciences, and \\ ${ }^{2}$ Molecular Medicine, 'Sapienza' University, Umberto I Hospital, I-00161 Rome; \\ ${ }^{3}$ CNR-IBPM, National Research Council, I-00161 Rome, Italy
}

Received June 18, 2013; Accepted July 25, 2013

DOI: $10.3892 / o r .2013 .2682$

\begin{abstract}
The aim of the present study was to evaluate human epididymis protein 4 (HE4) as a marker of epithelial ovarian cancer (EOC) relapse and the combination of this biomarker with contrast-enhanced high-resolution multidetector row computed tomography CE CT imaging to impove the monitoring of EOC patients. Twenty-one patients with advanced EOC (FIGO III/IV) who underwent surgery and adjuvant chemotherapy were retrospectively selected. Each patient contributed 3 serum samples drawn at 3-month intervals: time interval I (1-3 months from surgery), time interval II (4-6 months from surgery) and time interval III (7-10 months from surgery). Serum HE4 and cancer antigen-125 (CA-125) levels were determined by EIA and IRMA assays, respectively. Nine out of the 21 (Group A) women had disease relapse while 12 out of the 21 (Group B) women had stable disease during the follow-up study. Twenty out of the 21 patients underwent at least two CE CT follow-ups with an interval time of $\sim 6$ months. One patient did not undergo a second CE CT. In patients with relapsed EOC, an increase in HE4 was noted in 22,78 and $89 \%$ of patients within the time intervals I, II and III, respectively. Positivity for CA-125 was found later at time interval III and only in $44 \%$ of patients. Conversely, for EOC patients in remission, increase over the threshold level was observed only for marker CA-125 (4/12). The evaluation of imaging findings at interval time II showed a significant correlation with high levels of HE4 in 6 out of 9 patients with recurrent disease. This study supports the hypothesis that HE4 may serve as an early biomarker for recurrence of EOC.
\end{abstract}

Correspondence to: Dr Emanuela Anastasi, Laboratory of Tumor Markers, Department of Molecular Medicine, 'Sapienza' University, Viale Regina Elena 324, I-00161 Rome, Italy

E-mail: emanuela.anastasi@uniroma1.it

*Contributed equally

Key words: biomarker, human epididymis protein 4, cancer antigen-125, CE CT, epithelial ovarian cancer
Moreover, HE4 serum levels combined with CE CT imaging may improve the monitoring management of women affected by ovarian cancer.

\section{Introduction}

Epithelial ovarian cancer (EOC) is the deadliest gynecological malignancy since approximately $75 \%$ of ovarian cancer cases present at an advanced stage when the disease has spread well beyond the ovaries and the cancer involves the peritoneal cavity or other organs (1). The cancer is insidious; frequently initial symptoms occur only during the advanced stage of the disease and are often related to the presence of a grossly enlarged tumor and extensive ascites fluid (2).

According to a report of the American Joint Committee on Cancer (AJCC), patients with stage III and IV EOC have 5-year relative survival rates of 33.5 and $17.9 \%$. In contrast, patients with stage I and II disease have 5-year relative survival rates of 89.3 and $65.5 \%$, respectively (3).

This disparity in survival between early and late stage disease emphasizes the need to improve early detection and diagnosis of EOC. Effective screening protocols are not currently available, and risk assessment for the presence of EOC in women with an ovarian cyst needs improvement.

The role of tumor biomarkers in the diagnosis and follow-up of ovarian carcinoma is a controversial issue. During the last several decades, several tumor markers have been detected in the blood of patients with EOC, yet their sensitivities and specificities for predicting this form of cancer appear no better than those of mucin CA-125 alone $(4,5)$.

The cancer antigen-125 (CA-125) is a glycoprotein belonging to the family of mucins encoded by the gene MUC16. The increased expression of this molecule is often associated with benign gynecological pathologies, and with non-gynecological conditions such as chronic liver disease, pancreatitis, kidney disease and chronic inflammatory diseases $(5,6)$. Because of the poor sensitivity and specificity of CA-125, in recent years, research has focused on the identification of new biomarkers that can provide higher sensitivities 
and specificities. Human epididymis protein 4 (HE4) has been recently accepted by the US Food and Drug Administration (FDA) as a monitoring marker for the management of patients with EOC. This new molecule, encoded by the gene WFDC2, is a glycoprotein of $20-25 \mathrm{kDa}$ initially identified in the epithelium of the distal epididymis as a human protease inhibitor. Moreover, it was found to be involved in the maturation of sperm (7).

Several studies have demonstrated that HE4 is increased in EOC but not in ovarian tissue under normal conditions $(8,9)$. Recently, it has been proposed that the combined use of novel biomarkers such as HE4 and CA-125 improves the sensitivity and specificity when compared with either biomarker alone for the diagnosis and management of EOC (10-12). Although several studies have focused on markers to facilitate the early diagnosis of disease; it is just as important to identify markers able to predict disease remission, response to therapy and, in particular, early detection of disease relapse.

Recurrent EOC, unlike other solid tumors, tends to present without accompanying symptoms and forms multiple small implantations, particularly in the small intestine and mesentery, which cannot be readily detected using conventional imaging techniques $(13,14)$.

Computed tomography (CT) has low sensitivity for detecting disease recurrence, probably due to its inability to detect small peritoneal implants and normal-sized lymph node metastases $(15,16)$. Moreover a number of patients with disease relapse can present with normal CA-125 levels $(15,17)$.

In the present study, we retrospectively investigated the expression of two markers, CA-125 and HE4, as indicators of relapse in EOC patients with recurrent disease in combination with contrast-enhanced high-resolution multidetector row computed tomography (CE CT) findings. Moreover, to improve the management of these patients we evaluated a possible correlation between HE4 levels and CE CT imaging results.

\section{Materials and methods}

Patients. All subjects included in the follow-up study were patients referred to the Oncologic Unit A, of the Policlinico Umberto I, Rome, Italy, from January 2009 to December 2011. Of the total 21 women, 18 were in a postmenopausal state (age range $50-85$ years, mean age $65.72 \pm 2.32$ ) and 3 patients were in a premenopausal state (age range $43-47$; mean age $44.73 \pm 1.20$ ). All patients had advanced EOC (FIGO III/IV), and underwent surgery and adjuvant chemotherapy, and were retrospectively selected for the assessment of HE4 and CA-125 levels. Sixteen out of the $21(76 \%)$ women were affected by papillary serous carcinoma and 5 out of 21 (24\%) were affected by papillary serous carcinoma 'poorly' differentiated and undifferentiated of grade G3/4. Each patient contributed 3 serum samples drawn at 3-month intervals as follows: time interval I (1-3 months from surgery), time interval II (4-6 months from surgery), time interval III (7-10 months from surgery).

Inclusion criteria for enrollment included that: all patients had to show clinical remission after surgery and undergo adjuvant chemotherapy. Nine out of the 21 women showed disease relapse during the follow-up study (Group A), 12 out of 21 women had stable disease during the follow-up study (Group B).
A written informed consent was obtained from all of the patients prior to the collection of blood samples.

Sample collection. Serum samples were collected in a red-top vacutainer following a standard protocol. Samples were clotted for 60-90 min, and centrifuged for $10 \mathrm{~min}$ at $1,300 \mathrm{x} \mathrm{g}$. The serum fractions were aliquoted and stored at $-80^{\circ} \mathrm{C}$ until analysis.

\section{Measurements of the biomarkers}

HE4. HE4 levels were determined using the HE4 EIA assay (Fujirebio Diagnostics). The HE4 EIA is a solid phase, non-competitive immunoassay based upon the direct 'sandwich' technique using 2 monoclonal antibodies, 2H5 and 3D8, directed against 2 epitopes in the C-WFDC domain of HE4. Standard and control/patient serum samples were incubated with biotinylated anti-HE4 monoclonal antibody $2 \mathrm{H} 5$ aliquots in streptavidin-coated microstrips. HE4 in standard or serum samples was adsorbed in the streptavidin-coated microstrips by the biotinylated anti-HE4 monoclonal antibody during the incubation period. The strips were then washed and incubated with HRP-labeled anti-HE4 monoclonal antibody 3D8. After washing, buffered substrate/chromogen reagent was added to each strip, and the enzyme reaction was able to proceed. During the enzyme reaction, a blue color developed when the antigen was present. The intensity of the color was directly proportional to the amount of HE4 present in the samples. According to the manufacturer's indications, normal values of HE4 were considered to be $<150 \mathrm{pmol} / \mathrm{l}$.

CA-125.CA-125 levels were evaluated by a one-step 'sandwich' radioimmunoassay (Radim, The Netherlands). Polystyrene beads coated with the M11 capture antibody reacting with molecules containing OC 125 reactive determinants were incubated with the control or patient serum samples, standards, and tracer ( ${ }^{125}$ I-labeled mouse monoclonal OC 125 antibody) aliquots. The bound radioactivity observed was proportional to the concentration of the OC 125 reactive determinant (antigen). Normal levels of CA-125 were considered to be $<35 \mathrm{U} / \mathrm{ml}$.

Imaging. During the study period, 20 out of 21 patients underwent at least two CE CT follow-ups with an time interval of $\sim 6$ months. For 2 out of 21 cases, a third CE CT was performed within 3 months from the previous one, due to a worsening in the patient clinical conditions. The second CE CT generally was carried out within the time interval II of the serum sampling. In 1 patient (Group A) a second CE CT was not performed.

Contrast-enhanced high-resolution multidetector row computed tomography (Somatom Sensation 64; Siemens Medical System, Erlangen, Germany) was used to evaluate peritoneal carcinomatosis. The parameters applied were as follows: $0.6 \times 64 \mathrm{~mm}^{2}$ collimation, $3-\mathrm{mm}$ section thickness, 250 effective $\mathrm{mAs}, 120 \mathrm{kVp}$ and $0.8-1.5 \mathrm{~mm}$ reconstruction interval of coronal and sagittal images. The CT scans were acquired in basal conditions and after administration of contrast medium, cranio-caudally, from the dome of the diaphragm to the pelvis. The images were post-processed [multiplanar reconstruction (MPR) and maximum intensity 
Table I. Levels of HE4 and CA-125 determined in patients with disease relapse (Group A) and stable disease (Group B) within 3 time ranges.

\begin{tabular}{|c|c|c|c|c|}
\hline & \multicolumn{2}{|c|}{ Group A ( $n=9)$, median (range) } & \multicolumn{2}{|c|}{ Group B $(n=12)$, median (range) } \\
\hline & HE4 (pmol/l) & CA-125 (U/ml) & HE4 (pmol/l) & CA-125 (U/ml) \\
\hline Time interval I & $120(90-160)^{\mathrm{a}}$ & $15(10-30)$ & $55.5(40-135)^{\mathrm{a}}$ & $13(8-56)$ \\
\hline Time interval II & $165(97-241)^{b}$ & $20(11-30)^{\mathrm{d}}$ & $63(30-132)^{\mathrm{b}}$ & $11.5(8-86)^{\mathrm{d}}$ \\
\hline Time interval III & $300(137-806)^{\mathrm{c}}$ & $34(12-170)^{\mathrm{e}}$ & $55(30-126)^{\mathrm{c}}$ & $12(8-170)^{\mathrm{e}}$ \\
\hline
\end{tabular}

${ }^{\mathrm{a}} \mathrm{p}<0.002 ;{ }^{\mathrm{b}} \mathrm{p}<0.0004 ;{ }^{\mathrm{c}} \mathrm{p}<0.0001 ;{ }^{\mathrm{d}} \mathrm{p}<0.02 ;{ }^{\mathrm{e}} \mathrm{p}<0.02$. HE4, human epididymis protein 4 ; CA-125, cancer antigen-125. Time interval I (1-3 months following surgery). Time interval II (4-6 months following surgery). Time interval III (7-10 months following surgery).

projection (MIP)] with reconstructions in sagittal and coronal plane sections with a 1-mm interval to improve the anatomical analysis, particularly of the surface lesions.

An intravenous injection of $20 \mathrm{mg}$ of butyl-scopolamine (Buscopan; Boehringer) was administered to all patients to relax the bowel wall and reduce peristalsic bowel movement before CT examinations. The patients were asked to ingest 1 liter of water in order to achieve optimal distension of the stomach and of the bowel.

A dual phase protocol, arterial and venous phase, after intravenous administration of contrast medium, was performed in order to assess local tumor extent. Nonionic iodinated contrast medium (350 mg I/ml Iomeron; Bracco, Milan, Italy) was administered intravenously utilizing an automatic injector (Stellant DCT; Medrad, Warrendale, PA, USA) at a rate of infusion of 3-3.5 ml/sec for a total volume of 90-120 ml.

Parameters of tumor extension, i.e. peritoneal carcinomatosis and lymph node dissemination, were evaluated according to an arbitrary scoring classification as follows. Peritoneal carcinomatosis: score 0 , undetectable carcinomatosis; score 1 , single or multiple sites of micro-nodular implants $(<1 \mathrm{~cm})$ above or below the mesocolon; score 2, diffuse macro-nodular implants $(>1.5 \mathrm{~cm})$ on the bowel surface or mesenterial implants, and omental involvement with marked thickening (omental cake). Lymph node dissemination: L0, lymph nodes 0.5-1 cm diameter; L1, lymph nodes $1-1.5 \mathrm{~cm}$; L2, lymph nodes $1.5-2.5 \mathrm{~cm}$; L3, clusters of lymph nodes $>2.5 \mathrm{~cm}$ diameter.

Finally, we compared the two CE CT performed during the study period for each patient in order to determine disease progression, remission or stable disease.

Progression of disease was diagnosed when the comparison between the two CE CT showed an increase in the score (carcinomatosis and/or lymph node) or an increase in the size and/or number by $>20 \%$ of at least 3 implants of carcinomatosis defined as target lesions.

Remission of disease was considered when there was either a decrease in the score (carcinomatosis and/or lymph node), or a decrease in the size and/or number by $30 \%$ of the target lesions.

Stability was indicated when the disease did not follow any of the parameters described above.

Statistical analysis. For each patient, the mean age \pm SEM and the median (range) of serum HE4 and CA-125 levels were determined. Box plots were generated for each marker in Group A and B patients. Statistically significant differences were assessed by the nonparametric Mann-Whitney test for categorical variables using MedCalc ${ }^{\circledR}$ v12.3.0 software. The level of statistical significance was set at $\mathrm{p}<0.05$.

\section{Results}

Biomarkers in the follow-up study. The concentrations of HE4 and CA-125, expressed as median and range, measured in Group A and B within time intervals I, II and III, are provided in Table I.

Group A. In the 9 patients who showed disease recurrence, during the follow-up study, absolute concentrations of HE4 above the threshold of positivity ( $150 \mathrm{pmol} / \mathrm{l})$ were noted in 22,78 and $89 \%$ of patients within time intervals I, II and III following surgery, respectively.

We also observed that the serum levels of CA-125 were consistently below the threshold value $(35 \mathrm{U} / \mathrm{ml})$ during time intervals I and II, while only $44 \%$ of patients had positive absolute concentrations of the marker in the time interval III (Fig. 1). In these patients, the increase in HE4 level above the cut-off preceded the rise of CA-125 3 months. Furthermore, in the last time window, the mean increase of HE4 was $~ 3$-times the cut-off while the mean increase in CA-125 was $\sim 1.5$-times the value of positivity (Fig. 2).

Group B. In the 12 patients who had stable disease, the HE4 concentrations were within the range of normality for all determinations, while for CA-125, 6 positive values were present in 4 different patients (Fig. 3).

The median serum levels of HE4, drawn at 3-month intervals, were significantly higher in Group A when compared with values in Group B patients (time interval I: $120 \mathrm{vs} .55 .5 \mathrm{pmol} / \mathrm{l}$ p<0.002; time interval II: 165 vs. 63 pmol/l, p<0.0004; time interval III: 300 vs. 55 pmol/1, p<0.0001). The median serum levels of CA-125 measured between the 2 groups of women, were statistically different only during time intervals II and III (20 vs. $11.5 \mathrm{U} / \mathrm{ml}, \mathrm{p}<0.02$ and 34 vs. $12 \mathrm{U} / \mathrm{ml}, \mathrm{p}<0.02$, respectively).

\section{Imaging in the follow-up study}

Group A. In 1 out of 9 patients in Group A, the first CT showed no visible carcinomatosis (score 0 ) the second CE CT showed the presence of multiple implants smaller than $1.5 \mathrm{~mm}$ 

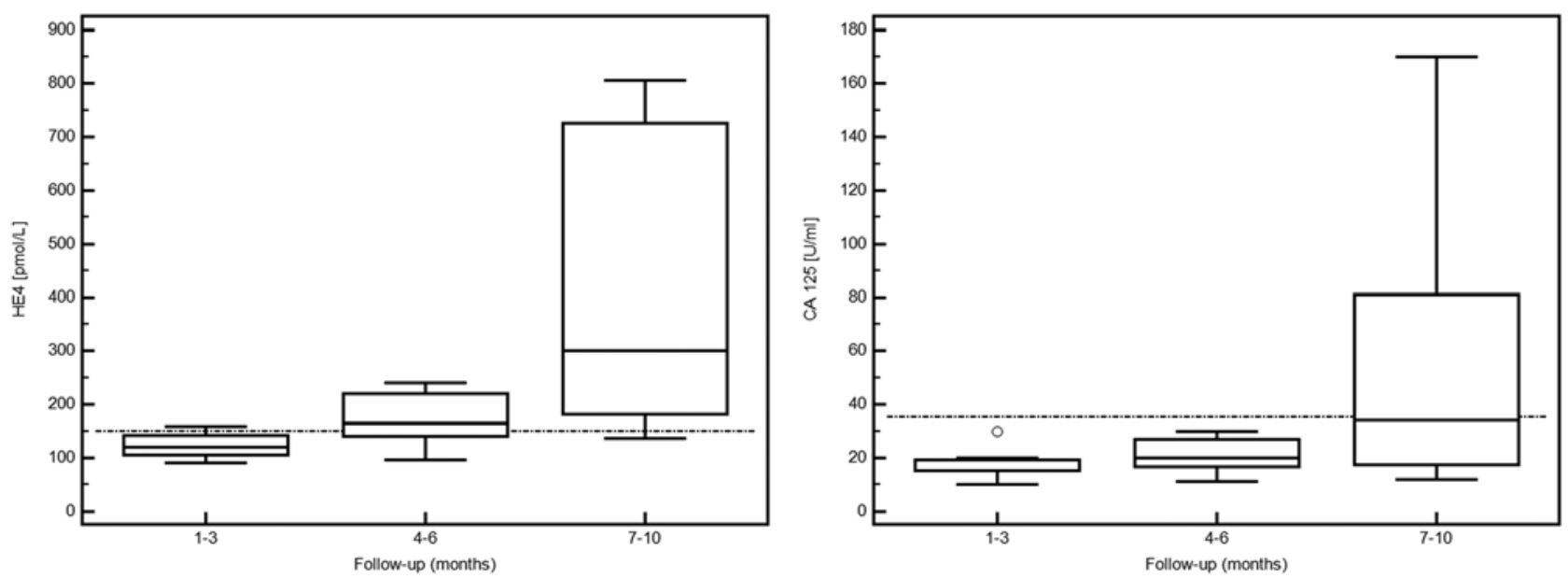

Figure 1. Distribution of HE4 and CA-125 levels in patients with recurrent disease (Group A). The box plots represent the median values and 25-75th percentiles for both markers. HE4, human epididymis protein 4; CA-125, cancer antigen-125.

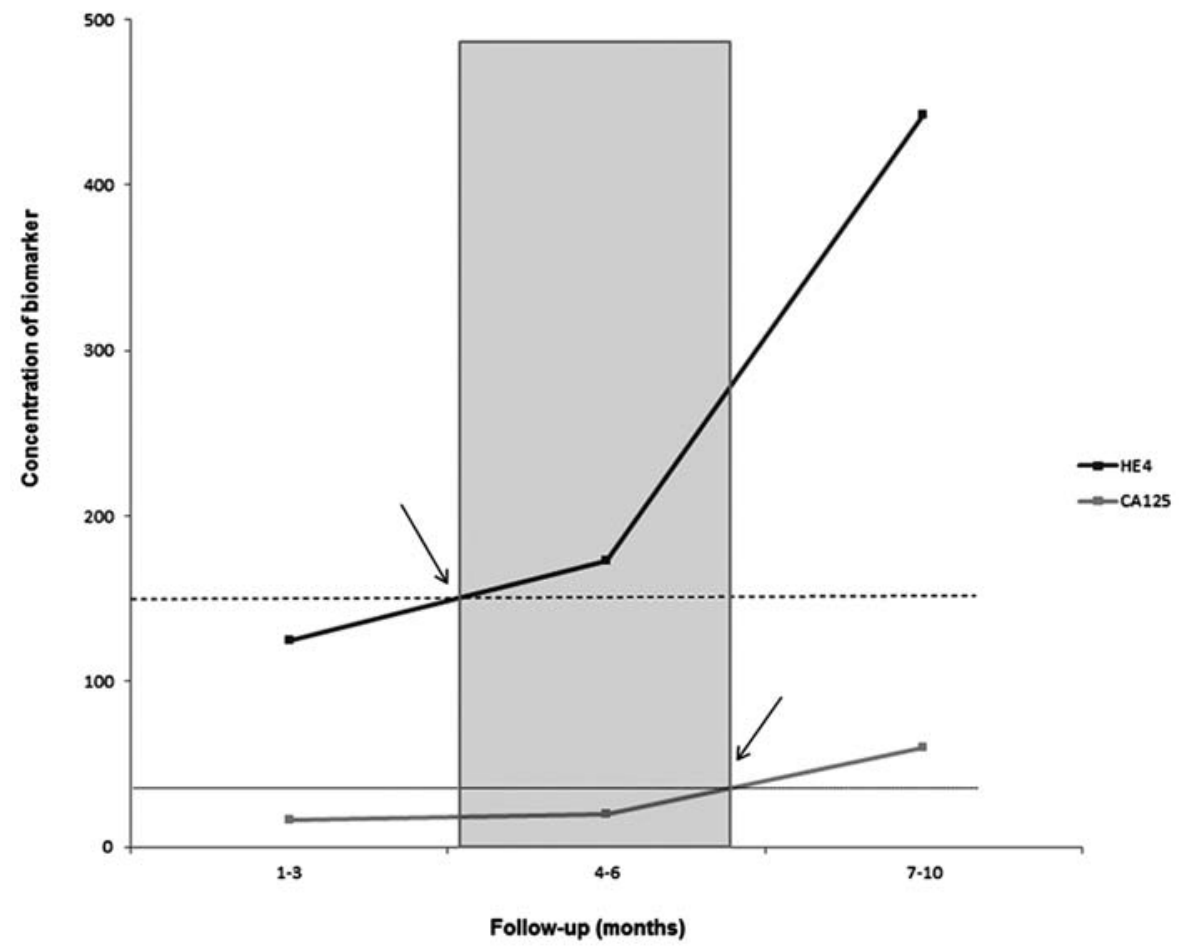

Figure 2. Trend of the average values of HE4 and CA-125 in Group A women. The area of the rectangle represents graphically the temporal gain anticipation of HE4 compared to CA-125. The threshold values of positivity for HE4 $(150 \mathrm{pmol} / \mathrm{l})$ and CA-125 (35 U/ml) are represented by the dashed (upper) and solid (lower) line, respectively. HE4, human epididymis protein 4; CA-125, cancer antigen-125.

(score 1). Moreover, a worsening of clinical condition justified a third examination ( 3 months after the second $\mathrm{CT}$ ) which showed severe progression of disease (score 2 , lymph nodes 3 ) with associated ascites (Fig. 4).

Six out of the 9 patients in Group A had an initial condition (first CT) of score 1 and lymph nodes 1 or 2 . In 5 out of 6 of these cases the second CE CT showed progressive disease with an increased score both for carcinomatosis and lymph nodal disease. The remaining case had stable disease at the second CE CT, while a third examination was performed after a worsening condition, and the CE CT determined disease progression as well.
Finally only 1 out of 9 patients, showed an initial condition of score 2 and L2, which was determined to be L3 disease at the second CE CT with increased size and number of carcinomatosis implants.

Group B. In 10 out of 12 cases, the first CE CT showed no visible carcinomatosis implants (score 0 ), and there was no image worsening at the second CE CT during the follow-up.

In 2 out of 12 patients in Group B, the first CE CT showed an initial condition of score 1 and lymph nodes 1 ; the second CE CT showed a decrease in carcinomatosis implants which were no longer visible (score 0) (Fig. 5). 

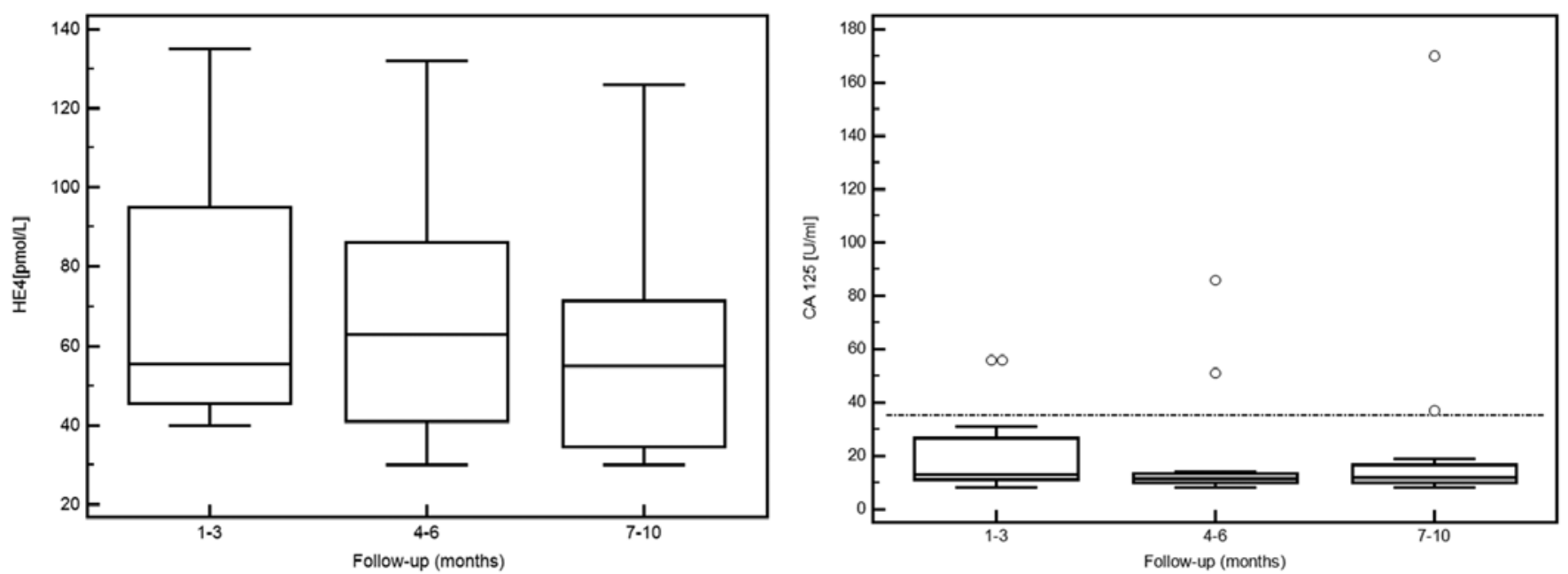

Figure 3. Distribution of HE4 and CA-125 levels in patients with stable disease (Group B). The box plots represent the median values and 25-75th percentiles for both markers. HE4, human epididymis protein 4; CA-125, cancer antigen-125.

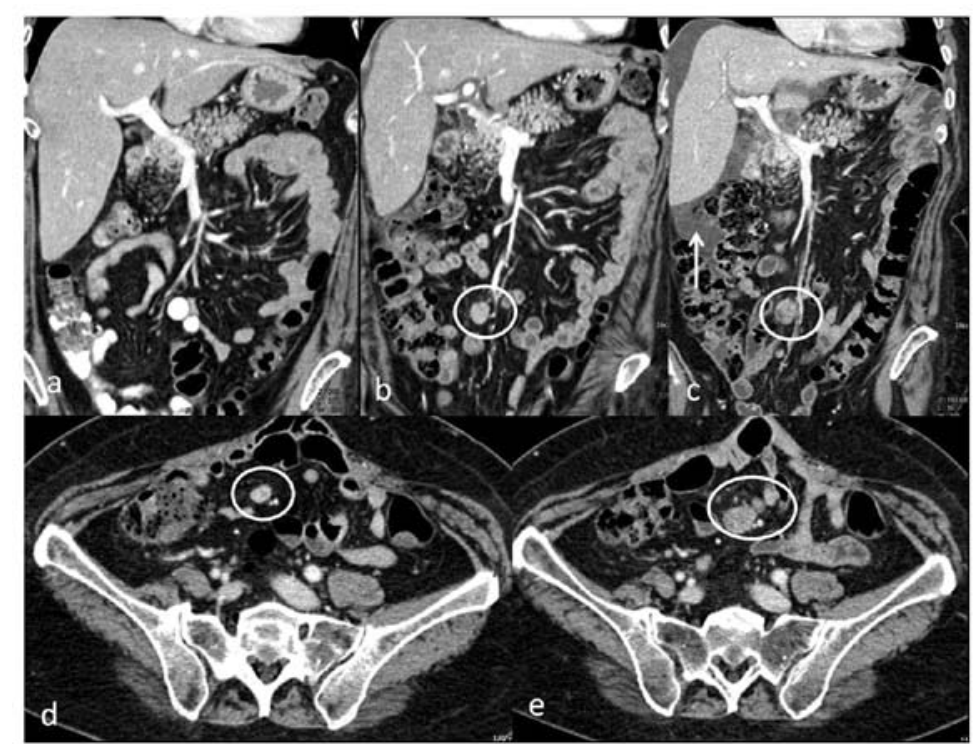

Figure 4. Patient with disease progression (Group A). The images, in coronal plane reconstruction CT, are at different times: (a) initial CT; (b) 6 months later and (c) 9 months later. The images in the axial plane ( $\mathrm{d}$ and e) confirm the recurrence of the disease. The circles indicate the carcinomatosis nodules which were absent in the first CT. The last CT (c) also shows peritoneal effusion (white arrow) which is also a sign of disease progression. CT, computed tomography.

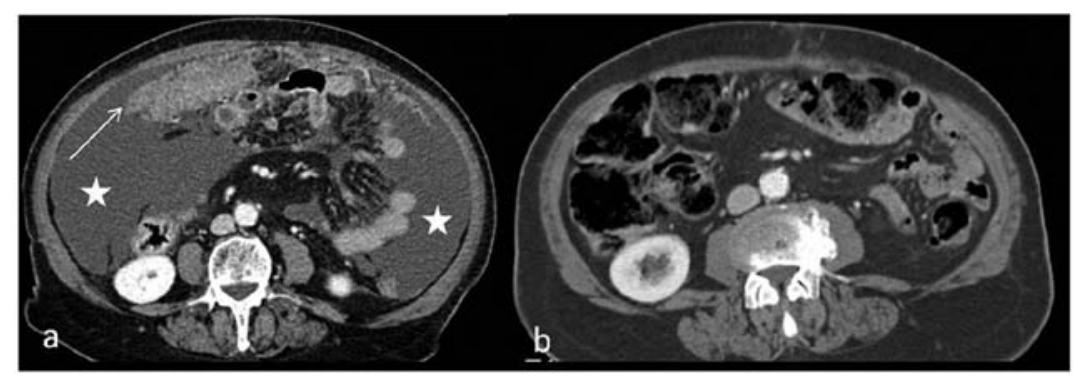

Figure 5. Patient with disease remission (Group B). The two images show the same axial CT plane taken (a) at initial CT and (b) 6 months later. The white arrow in panel a shows a severe peritoneal carcinomatosis associated with ascites, in $\mathrm{b}$ the image shows no signs of peritoneal carcinomatosis.

Imaging and biomarkers. The evaluation of the imaging findings within time interval II, which generally corresponded to the time of the second CE CT, showed that Group A women presented with peritoneal carcinomatosis and lymph node 
dissemination with various degrees of disease progression. In particular, 6 out of 9 patients showed elevated HE4 levels corresponding with widespread macro-nodular implants $(>1.5 \mathrm{~cm})$ on the bowel or mesenterial surface (score 2) and lymph nodal disease $\mathrm{L} 2 / \mathrm{L} 3$.

The HE4 results of the Group B women were in agreement with undetectable carcinomatosis (score 0 ), small lymph node size without radiological significance (L1/L0). Furthermore, CA-125 levels did not correlate with the different degrees of carcinomatosis in both Group A and B.

\section{Discussion}

New and innovative approaches are needed for the early detection of disease relapse in women affected by epithelial ovarian cancer (EOC). Regardless of the development of new treatments and therapies designed to improve the 5-year survival rate, ovarian cancer still remains the deadliest type of cancer of the female reproductive tract.

Biomarkers have great potential to serve as an efficient screening tool for the early detection of ovarian cancer (1). CA-125 is still the only tumor marker recommended as a diagnostic or prognostic indicator and for the monitoring of disease recurrence after surgery and adjuvant chemotherapy (18-20). The major drawback of CA-125 is the documented lack of specificity, as this marker may show levels exceeding the 95th percentile of normal values in a significant proportion of women with benign or malignant diseases (21). Accordingly, there have been many efforts to improve the diagnostic performance of CA-125. Among these, relevance has recently been given to HE4 which is one of the most promising markers with improved sensitivity and specificity. Moreover, for clinical management of patients affected by EOC, it is of paramount importance to identify markers able to predict early relapse of clinical disease during follow-up.

Numerous studies have shown that HE4 is able to anticipate the relapse of disease compared to CA-125 $(11,22)$. Based on these data we conducted a retrospective follow-up study of 10 months. We enrolled 21 patients diagnosed with ovarian cancer stages FIGO III/IV, who underwent medical or surgical treatment. Of great importance from the clinical point of view, are the results obtained in 9 out of 21 patients who exhibited disease recurrence during the study period. In particular, during time interval II (4-6 months following surgery) 78\% of the patients had positive values for HE4 while, at the same time, none showed alteration in serum CA-125. It is important to note that the positivity for CA- 125 shown in $44 \%$ of patients occurred only during the last time window of our study (7-10 months). Therefore, we conclude that, in case of disease recurrence, increased levels of HE4 may precede an elevation in CA- 125 by $\sim 3$ months.

Our results are in agreement with literature data which suggest that the biomarker CA-125 cannot reliably detect small tumor masses $<8 \mathrm{~cm}^{3}$ (23). Thus, CA-125 is not suitable as an indicator for the presence of microscopic minimal residual disease following radical surgery and/or chemotherapy (24).

Furthermore, a randomized (EORTC 55955) trial showed that there is no survival benefit for early treatment based on increased CA-125 levels alone $(25,26)$. This may agree with the evidence suggesting that this marker is increased when the disease is already widespread.

In the present study, we used CE CT to assess the recurrence of disease, and correlated the findings with tumor progression or stable disease with serum levels of the biomarkers.

In the last decade, several studies have demonstrated the important role of CE CT as an investigation of choice in both pre-operative staging and follow-up (27), due to the short time taken, the good cost-effective ratio and the widespread availability of the technique.

It is widely accepted that, in preoperative staging, CE CT allows confirmation of the presence of a malignant adnexal mass, as well as allows the determination of the extent of the disease. Both features are crucial for treatment planning. Moreover, CE CT has proven to be useful for assessment of the treatment response, and to diagnose disease recurrence. In this regard CE CT was demonstrated to have a sensitivity of $85-95 \%$ in the detection of disease recurrence (28).

However, it is necessary to specify that several studies are currently comparing the use of CE CT and PET/CT as surveillance tools for recurrent disease. In fact, the main limitation of CE CT is the reduced sensitivity $(\sim 50 \%)$ for the detection of peritoneal implants $<10 \mathrm{~mm}$ (28), or for the differentiation of these from fibrous residual after treatment. However, there are no reliable data which favor PET/CT rather than CE CT and vice versa (29).

In light of these data, it is necessary to combine imaging data with clinical data, such as serial measurements of tumor biomarkers for an early detection of recurrent disease. In the present study, regarding disease recurrence or stable disease, CE CT findings correlated with the serum level of HE4. In particular, evaluation of the imaging results during time interval II (4-6 months following surgery) showed a significant correlation with high levels of HE4 in 6 out of 9 patients with recurrent disease. Moreover the HE4 results of the women with stable disease correlated with undetectable carcinomatosis (score 0 ) and with small lymph node size without radiological significance (L1/L0).

These data indicate that HE4 is superior to CA-125 for estimating the extent of peritoneal carcinomatosis and that it correlates with tumor burden in all surgically treated patients (30-32).

In conclusion, this follow-up study supports the hypothesis that HE4 may be an early biomarker for the recurrence of EOC and that the HE4 serum levels, combined with CE CT imaging, could improve the monitoring management of women affected by this tumor. However, it could have enormous benefits on patient life. In fact, this may justify the use of this marker for a strict follow-up rather than excessive use of CE CT imaging, which may be needed in case of a positive value, considering the side-effects due to ionizing radiation and injection of contrast medium associated with this technique. It is obvious that our findings warrant further validation.

\section{Acknowledgements}

The authors are thankful to Giuseppina Gennarini, Barbara Colaprisca and Silvestra Tudini for their excellent technical assistance. 


\section{References}

1. Kobayashi E, Ueda Y, Matsuzaki S, Yokoyama T, Kimura T, Yoshino K, Fujita M, Kimura T and Enomoto T: Biomarkers for screening, diagnosis, and monitoring of ovarian cancer. Cancer Epidemiol Biomarkers Prev 21: 1902-1912, 2012.

2. Berek JS, Crum C and Friedlander M: Cancer of the ovary, fallopian tube, and peritoneum. Int J Gynaecol Obstet 119 (Suppl 2): S118-S129, 2012.

3. Husseinzadeh N: Status of tumor markers in epithelial ovarian cancer has there been any progress? A review. Gynecol Oncol 120: $152-157,2011$.

4. Rosen DG, Wang L, Atkinson JN, Yu Y, Lu KH, Diamandis EP, Hellstrom I, Mok SC, Liu J and Bast RC Jr: Potential markers that complement expression of CA125 in epithelial ovarian cancer. Gynecol Oncol 99: 267-277, 2005.

5. Bast RC Jr, Badgwell D, Lu Z, et al: New tumor markers: CA125 and beyond. Int J Gynecol Cancer 15 (Suppl 3): 274-281, 2005.

6. Medeiros LR, Rosa DD, da Rosa MI, et al: Accuracy of CA 125 in the diagnosis of ovarian tumors: a quantitative systematic review. Eur J Obstet Gynecol Reprod Biol 142: 99-105, 2009.

7. Kirchhoff C: Molecular characterization of epididymal proteins. Rev Reprod 3: 86-95, 1998.

8. Galgano MT, Hampton GM and Frierson HF Jr: Comprehensive analysis of HE4 expression in normal and malignant human tissues. Mod Pathol 19: 847-853, 2006.

9. Hellström I, Raycraft J, Hayden-Ledbetter M, et al: The HE4 (WFDC2) protein is a biomarker for ovarian carcinoma. Cancer Res 63: 3695-3700, 2003.

10. Li J, Dowdy S, Tipton T, Podratz K, Lu WG, Xie X and Jiang SW: HE4 as a biomarker for ovarian and endometrial cancer management. Expert Rev Mol Diagn 9: 555-566, 2009.

11. Anastasi E, Marchei GG, Viggiani V, et al: HE4: a new potential early biomarker for the recurrence of ovarian cancer. Tumour Biol 31: 113-119, 2010.

12. Moore RG and Maclaughlan S: Current clinical use of biomarkers for epithelial ovarian cancer. Curr Opin Oncol 22: 492-497, 2010

13. Tempany CM, Zou KH, Silverman SG, Brown DL, Kurtz AB and McNeil BJ: Staging of advanced ovarian cancer: comparison of imaging modalities - report from the Radiological Diagnostic Oncology Group. Radiology 215: 761-767, 2000.

14. Gronlund B, Høgdall C, Hilden J, Engelholm SA, Høgdall EV and Hansen HH: Should CA-125 response criteria be preferred to Response Evaluation Criteria In Solid Tumors (RECIST) for prognostication during second-line chemotherapy of ovarian carcinoma? J Clin Oncol 22: 4051-4058, 2004.

15. Gu P, Pan LL, Wu SQ, et al: CA 125, PET alone, PET-CT, CT and MRI in diagnosing recurrent ovarian carcinoma: a systematic review and meta-analysis. Eur J Radiol 71: 164-174, 2009.

16. Togashi K: Ovarian cancer: the clinical role of US, CT, and MRI. Eur Radiol 13 (Suppl 4): L87-L104, 2003.

17. Goonewardene TI, Hall MR and Rustin GJ: Management of asymptomatic patients on follow-up for ovarian cancer with rising CA-125 concentrations. Lancet Oncol 8: 813-821, 2007.

18. Aebi S and Castiglione M; ESMO Guidelines Working Group: Newly and relapsed epithelial ovarian carcinoma: ESMO clinical recommendations for diagnosis, treatment and follow-up. Ann Oncol 20 (Suppl 4): 21-23, 2009.
19. Sturgeon CM, Duffy MJ, Stenman U-H, et al: National Academy of Clinical Biochemistry laboratory medicine practice guidelines for use of tumor markers in testicular, prostate, colorectal, breast, and ovarian cancers. Clin Chem 54: e11-e79, 2008.

20. Bast RC Jr: Status of tumor markers in ovarian cancer screening. J Clin Oncol 21 (Suppl 10): s200-s205, 2003.

21. Moore RG, Jaube-Raughley M, Brown AK, et al: Comparison of a novel multiple marker assay vs. the Risk of Malignancy Index for the prediction of epithelial ovarian cancer in patients with a pelvic mass. Am J Obstet Gynecol 203: e1-e6, 2010.

22. Schummer M, Drescher C, Forrest R, Gough S, Thorpe J, Hellström I, Hellström KE and Urban N: Evaluation of ovarian cancer remission markers HE4, MMP7 and Mesothelin by comparison to the established marker CA125. Gynecol Oncol 125: 65-69, 2012.

23. Hori SS and Gambhir SS: Mathematical model identifies blood biomarker-based early cancer detection strategies and limitations. Sci Transl Med 3: 109ra116, 2011.

24. Häfner N, Nicolaus K, Weiss S, Frey M, Diebolder H, Rengsberger M, Dürst M and Runnebaum IB: p53-autoantibody may be more sensitive than CA-125 in monitoring microscopic and macroscopic residual disease after primary therapy for epithelial ovarian cancer. J Cancer Res Clin Oncol 139: 1207-1210, 2013

25. Rustin GJ: Follow-up with CA125 after primary therapy of advanced ovarian cancer has major implications for treatment outcome and trial performances and should not be routinely performed. Ann Oncol 22 (Suppl 8): viii45-viii48, 2011.

26. Rustin GJ, van der Burg ME, Griffin CL, et al: Early versus delayed treatment of relapsed ovarian cancer (MRC OV05/EORTC 55955): a randomised trial. Lancet 376: 1155-1163, 2010.

27. Forstner R, Sala E, Kinkel K and Spencer JA; European Society of Urogenital Radiology: ESUR guidelines: ovarian cancer staging and follow-up. Eur Radiol 20: 2773-2780, 2010.

28. Coakley FV, Choi PH, Gougoutas CA, Pothuri B, Venkatraman E, Chi D, Bergman A and Hricak H: Peritoneal metastases: detection with spiral CT in patients with ovarian cancer. Radiology 223: 495-499, 2002.

29. Sala E, Kataoka M, Pandit-Taskar N, Ishill N, Mironov S Moskowitz CS, Mironov O, Collins MA, Chi DS, Larson S and Hricak H: Recurrent ovarian cancer: use of contrast-enhanced $\mathrm{CT}$ and PET/CT to accurately localize tumor recurrence and to predict patients' survival. Radiology 257: 125-134, 2010

30. Diniz Bizzo SM, Meira DD, Lima JM, Mororó Jda S, Casali-da-Rocha JC and Ornellas MH: Peritoneal VEGF burden as a predictor of cytoreductive surgery outcome in women with epithelial ovarian cancer. Int J Gynaecol Obstet 109: 113-117, 2010.

31. Kang S: The role of neoadjuvant chemotherapy in ovarian cancer patients with extensive tumor burden. J Gynecol Oncol 22: 299-300, 2011.

32. Midulla C, Manganaro L, Longo F, Viggiani V, Frati L, Granato T and Anastasi E: HE4 combined with MDCT imaging is a good marker in the evaluation of disease extension in advanced epithelial ovarian carcinoma. Tumour Biol 33: 1291-1298, 2012. 